\title{
Sulla unicità degli sviluppi delle funzioni di una variabile in serie di funzioni $\boldsymbol{X}_{\|}$.
}

\author{
APPENDICE ALLA MEMORIA PREGEDENTE
}

(del prof. Ulisse Dini, a Pisa.)

\begin{abstract}
A rendere più completi gli studî che ho fatto nella Memoria che precede intorno agli sviluppi delle funzioni di una 0 di due variabili in serie di funzioni $X_{n}$ e $Y_{n}$, occorrerebbe anche fare vedere che tali sviluppi non possono farsi che in un sol modo. Fino a questi ultimi tempi si era creduto di potere dimostrare questa proprietả per mezzo della integrazione per serie, ma la dimostrazione che se ne dava, quando non si facesse alcuna limitazionc intorno alla natura della serie, era tutt'altro che rigorosa. Io ho cercato perciò di dimostrare rigorosamente questa proprietà, almeno pel caso delle funzioni di una sola variabile, e ora sono giunto a dimostrare anche più in generale che «se $f(x)$ è una funzione di $x$ che fra -1 e 1 è sempre "determinata e finita o cessa di essere tale soltanto in un numero finito «di punti 0 in un gruppo infinito di punti di prima specie $\left(^{*}\right)$, non possono " esistere due serie distinte di funzioni $X_{n}, \sum a_{n} X_{n}, \sum a_{n}^{\prime} X_{n}$ che rappresen" tino la stessa funzione $f(x)$ per tutti i valori di $x$ fra -1 e 1 , o anche «soltanto pei valori di $x$ che restano nello stesso intervallo da -1 a 1
\end{abstract}

(*) Per la definizione e per le proprieta dei gruppi di punti puó vedersi la Memoria di Canton: Ueber die Ausdehnung eines Satzes aus der Theorie der trigonom. Reihen (Mathem. Annalen herausgeg. von Clebsch und Neumans, V. Band, p. 128), notando peró che io chiamo quì gruppi di punti i Punktmenge di Cantor, e gruppi di punti di prima specie quelli che hanno un numero finito $\vee$ di gruppi derivati abgeleitete Punktmengen), e che Cantor chiama Punktmenge von der ten Art. 
"quando si tolgono quelli corrispondenti a un numero finito di punti o a "un gruppo infinito di punti di prima specie»; e la mia dimostrazione è la seguente.

Incominciamo dall' osservare che, se esistessero le due serie qui dette $\boldsymbol{\Sigma} a_{n} X_{n}, \Sigma a_{n}^{\prime} X_{n}$, dovrebbe esistere una terza serie $\Sigma A_{n} X_{n}$ che per tutti i valori di $x$ fra -1 e 1 , eccettuati tutt'al più quelli corrispondenti a un numero finito di punti $o$ a un gruppo infinito di punti di prima specie, avessero sempre per somma zero, e i cui coefficienti $A_{n}$ non fossero tutti uguali a zero; e quindi pel teorema che vogliamo dimostrare basterà fare vedere che una tal serie non può esistere.

Ammettiamo percio che una tal serie possa esistere, e sia la serie $\Sigma A_{n} X_{n}$; si avrà $\sum A_{n} X_{n}=0$ per tutti i valori di $x$ da -1 a 1 , tranne tutt'al più per quelli corrispondenti a un numero finito di punti o a un gruppo infinito di punti di prima specie; quindi, escludendo questi punti singolari (se esistono) con un numero finito d'intervalli piccoli quanto si vuole $i_{1}, i_{2}, i_{3}, \ldots$, negli intervalli restanti $\lambda_{1}, \lambda_{2}, \ldots$ si avrà sempre $\Sigma A_{n} X_{n}=0$; e perciò in questi intervalli le quantità $A_{n} X_{n}$ col crescere indefinito di $n$ tenderanno sempre a zero.

Ora, ponendo $x=\cos \theta$, e indicando con $\varepsilon$ un numero differente da zero e positivo, piccolo quanto si vuole, per tutti i valori di $\theta$ fra $\varepsilon$ e $\pi-\varepsilon$ si avrà la formola seguente (vedi BONNET: Sur les développements des fonctions en séries ordonnées suivant les fonctions $X_{n}$ et $Y_{n}$; Journal de LiouviluE, ser. I, tom. XVII, pag. 2'70):

$$
X_{n}=\frac{2 \cos \left(\rho^{\theta}-\frac{\pi}{4}\right)}{\sqrt{2 n \pi \operatorname{sen} \theta}}\left(1+\frac{(-1)^{n}}{4 n}\right)+\frac{\cot \theta \operatorname{sen}\left(\rho^{\theta}-\frac{\pi}{4}\right)}{4 n \sqrt{2 n \pi \operatorname{sen} \theta}}+\frac{p_{n}}{n^{2} \sqrt{n}},
$$

ove $\rho=\frac{2 n+1}{2}$, e $p_{n}$ col crescere indefinito di $n$, restando però fisso $\varepsilon$, si mantiene sempre numericamente inferiore ad un numero finito ed è una funzione continua di $\theta$; quindi sarà :

$A_{n} X_{n}=\frac{A_{2}}{\sqrt{2 n \pi \operatorname{sen} \theta}}\left\{2 \cos \left(\rho \theta-\frac{\pi}{4}\right)\left(1+\frac{(-1)^{n}}{4 n}\right)+\frac{\cot \theta \operatorname{sen}\left(\rho \theta-\frac{\pi}{4}\right)}{4 n}+\frac{p_{n} \sqrt{2 \pi \operatorname{sen} \theta}}{n^{2}}\right\} ;$

e ora, siccome per quanto abbiamo detto sopra, in uno o più intervalli finiti fra $\varepsilon$ e $\pi-\varepsilon$ le quantità $A_{n} X_{n}$ tendono sempre a zero col crescere indefinitamente di $n$, ripetendo i ragionamenti fatti da $C_{A N T O R}$ nella sua Memoria: 
Ueber einen die trigonometrischen Reihen betreffenden Lehrsatz (Giornale di Borchardt, vol. 72, pag. 135 e seg.), coll'osservare anche che gli ultimi tre termini fra parentesi col crescere indefinito di $n$ tendono a zero, si concludera intanto che se $\mathrm{i}$ coefficienti $A_{n}$ non tendono essi pure a zero col crescere indefinito di $n(*)$, vi tenderanno però sempre le quantità $\frac{A_{n}}{\sqrt{n}}$.

Di qui risulta che la serie $\Sigma \frac{A_{n} p_{2} \operatorname{sen}^{\frac{3}{2} \theta}}{n^{2} \sqrt{n}}$ è convergente per tutti i valori di $\theta$ fra $\varepsilon$ e $\pi-\varepsilon$; e quindi in tutti gli intervalli $\mu_{1}, \mu_{2}, \ldots$ fra $\varepsilon$ e $\pi-\varepsilon$ che corrispondono agli antichi intervalli $\lambda_{1}, \lambda_{2}, \ldots$ nei quali non cadono punti singolari, si avrà sempre:

$\mathbf{\Sigma} \frac{A_{n}}{\sqrt{2 n \pi}}\left\{2 \operatorname{sen} \theta \cos \left(\rho \theta-\frac{\pi}{4}\right)\left(1+\frac{(-1)^{n}}{4 n}\right)+\frac{2 \cos \theta \operatorname{sen}\left(\rho \theta-\frac{\pi}{4}\right)}{8 n}\right\}+\sum \frac{A_{n} p_{n} \operatorname{sen}^{\stackrel{3}{3} \theta}}{n^{2} \sqrt{n}}=0$,

0 anche:

$$
\begin{gathered}
\sum \frac{A_{u}}{\sqrt{2 n \pi}}\left\{( 1 + \frac { ( - 1 ) ^ { n } } { 4 n } ) \left[\operatorname{sen}\left((\rho+1) \theta-\frac{\pi}{4}\right)-\operatorname{sen}\left((\rho-1) \theta-\frac{\pi}{4}\right) \mid+\right.\right. \\
\left.+\frac{\operatorname{sen}\left[(\rho+1) \theta-\frac{\pi}{4}\right]+\operatorname{sen}\left[(\rho-1) \theta-\frac{\pi}{4}\right]}{8 n}\right\}+\mathbf{\Sigma} \frac{A_{n} p_{n} \operatorname{sen}^{\frac{3}{2} \theta}}{n^{3} \sqrt{n}}=0,
\end{gathered}
$$

e percio in tutti gli intervalli $\mu_{1}, \mu_{2}, \ldots$ fra $\varepsilon$ e $\pi-\varepsilon$ la prima serie del primo membro sarà convergente, e avrà per somma una funzione $\phi(\theta)$ di $\theta$.

Ora, poichè le quantità $\frac{A_{n}}{\sqrt{n}}$ tendono a zero col crescere indefinito di $n$, la stessa serie si può evidentemente ridurre ad una serie trigonometrica:

$$
\Sigma \alpha_{n} \operatorname{sen}\left[(\rho+1) \theta-\frac{\pi}{4}\right]
$$

nella quale i coefficienti $\alpha_{n}$ tendono a zero col crescere indefinito di $n$; quindi, per un teorema dato da Riemann nella sua Memoria: Ueber die Darstellung einer Function durch eine trigonometrische Reihe (Göttingen, 1867) la serie:

(*) Questo accade sempre tutte le volte che uno almeno dei punti 1 e -1 non è un punto singolare, perchè allora una almeno delle due serie $\sum A_{n}, \sum(-1)^{n} A_{n}$ è convergente. 


$$
\begin{aligned}
-\sum \frac{A_{n}}{\sqrt{2 n \pi}} & \left\{\left(1+\frac{(-1)^{n}}{4 n}\right)\left[\frac{\operatorname{sen}\left[(\rho+1) \theta-\frac{\pi}{4}\right]}{(p+1)^{2}}-\frac{\operatorname{sen}\left[\left(p-1, \theta-\frac{\pi}{4}\right]\right.}{(p-1)^{2}}\right]+\right. \\
& \left.+\frac{1}{8 n}\left[\frac{\operatorname{sen}\left[(p+1) \theta-\frac{\pi}{4}\right]}{(p+1)^{2}}+\frac{\operatorname{sen}\left[(\rho-1) \theta-\frac{\pi}{4}\right]}{(p-1)^{3}}\right]\right\}
\end{aligned}
$$

rappresenterà una funzione $\phi_{1}(\theta)$ di $\theta$ finita e continua, che in ogni punto

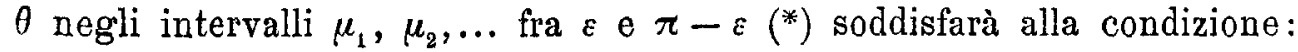

$$
\lim _{\alpha=0} \frac{\varphi_{1}(\theta+\alpha)-2 \varphi_{1}(\theta)+\varphi_{1}(\theta-\alpha)}{\alpha^{2}}=\phi(\theta)=-\sum \frac{A_{n} p_{n} \operatorname{sen}^{\frac{3}{2} \theta}}{n^{2} \sqrt{\pi}},
$$

e per tutti i punti $\theta$ fra $\varepsilon$ e $\pi-\varepsilon$ senza distinzione soddisfarà all'altra:

$$
\lim _{\alpha=0} \frac{\rho_{1}(\theta+\alpha)-2 \varphi_{1}(\theta)+\varphi_{1}(\theta-\alpha)}{\alpha}=0 \text {. }
$$

D'altra parte, siccome la serie $\mathbf{\Sigma} \frac{A_{n} p_{n} \operatorname{sen}^{\frac{3}{2} \theta}}{n^{2} \sqrt{n}}$ è anche convergente in ugual grado fra $\varepsilon$ e $\pi-\varepsilon$, se si pone $\psi(\theta)=\Sigma \frac{A_{n} p_{i z} \operatorname{sen}^{\frac{3}{2}} \theta}{n^{2} \sqrt{n}}, \psi(\theta)$ sarà una funzione finita e continua di $\theta$, e si avrà :

$$
\int_{\frac{\pi}{2}}^{\theta} d \theta \int_{\frac{\pi}{2}}^{\theta} \psi(\theta) d \theta=\mathbf{\Sigma} \frac{A_{n}}{n^{2} \sqrt{n}} \int_{\frac{\pi}{2}}^{\theta} d \theta \int_{\frac{\pi}{2}}^{1 \theta} p_{n} \operatorname{sen}^{\frac{3}{2}} \theta d \theta
$$

quindi fra $\varepsilon$ e $\pi-\varepsilon$ la serie del secondo membro sarà una funzione di $\theta$ finita e continua $\psi_{1}(\theta)$ che soddisfarà alle due condizioni:

$$
\begin{gathered}
\lim _{\alpha=0} \frac{\psi_{1}(\theta+\alpha)-2 \psi_{1}(\theta)+\psi_{1}(\theta-\alpha)}{\alpha^{2}}=\psi(\theta)=\sum \frac{A_{n} p_{n} \operatorname{sen}^{\frac{3}{2} \theta}}{n^{2} \sqrt{n}}, \\
\lim _{\alpha=0} \frac{\psi_{1}(\theta+\alpha)-2 \psi_{1}(\theta)+\psi_{1}(\theta-\alpha)}{\alpha}=0 ;
\end{gathered}
$$

(*) Qui parrebbe a prima vista che si dovessero escludere i punti $\varepsilon$ e $\pi-\varepsilon$ e gli estremi degli intervalli $\mu_{1}, \mu_{2}, \ldots$ quando anche questi intervalli vengono presi in considerazione. Peró, siccome il numero $\varepsilon$ può sempre impiccolirsi e gli intervalli $i_{1}, i_{2}, i_{3}, \ldots$ che escludono i punti singolari possono supporsi scelti in modo che possano prendersi anche intervalli più piccoli di quelli che qui abbiamo preso, s'intende subito che la indicata esclusione sarebbo per lo meno inutile. 
e conseguentemente la funzione $\phi_{1}(\theta)+\psi_{1}(\theta)$ sara una funzione $F(\theta)$ che fra $\varepsilon$ e $\pi-\varepsilon$ è sempre finita e continua e soddisfa alla condizione:

$$
\lim \frac{F(\theta+\alpha)-2 F(\theta)+F(\theta-\alpha)}{\alpha}=0
$$

e negli intervalli $\mu_{1}, \mu_{2}, \ldots$ fra $\varepsilon$ e $\pi-\varepsilon$ soddisfa altresì all'altra:

$$
\lim \frac{F(\theta+x)-2 F(\theta)+F(\eta-x)}{\alpha^{2}}=0 .
$$

Questo, per una osservazione fatta da CAnror nella Memoria che ho già citata in principio, porta subito a dire che la funzione $\left.F_{(} \theta\right)$ è una funzione di primo grado $c \theta+c^{\prime}$ in tutto l'intervallo fra $\varepsilon$ e $\pi-\varepsilon$; quindi nello stesso intervallo si avrà:

$$
\begin{gathered}
-\mathbf{\Sigma} \frac{A_{n}}{\sqrt{2 n \pi}}\left\{\left(1+\frac{(-1)^{n}}{4 n}\right)\left[\frac{\operatorname{sen}\left[(p+1)^{\theta}-\frac{\pi}{4}\right]}{(p+1)^{2}}-\frac{\operatorname{sen}\left[(p-1)^{\theta}-\frac{\pi}{4}\right]}{(p-1)^{2}}\right]+\right. \\
\left.+\frac{1}{8 n}\left[\frac{\operatorname{sen}\left[(p+1) \theta-\frac{\pi}{4}\right]}{(p+1)^{2}}+\frac{\operatorname{sen}\left[(p-1)^{\theta}-\frac{\pi}{4}\right]}{(p-1)^{2}}\right]\right\}+\sum \frac{A_{n}}{n^{2} \sqrt{n}} \int_{\frac{\pi}{2}}^{\theta} d 0 \int_{\frac{\pi}{2}}^{\theta} p_{n} \operatorname{sen}^{\frac{3}{4}} \theta d \theta=c \theta+c^{\prime},
\end{gathered}
$$

a alle serie del primo membro sarà anche applicabile una o più volte la integrazione definita in ogni intervallo fra $\varepsilon$ e $\pi-\varepsilon$, giacchè esse sono convergenti in ugual grado.

Ora, si ha evidentemente:

$$
\begin{aligned}
& \frac{\operatorname{sen}\left[(\rho+1) \theta-\frac{\pi}{4}\right]}{(\rho+1)^{2}}=-\int_{\frac{\pi}{2}}^{\theta} d \theta \int_{\frac{\pi}{2}}^{\theta} \operatorname{sen}\left[(\rho+1) \theta-\frac{\pi}{4}\right] d \theta+\frac{\operatorname{sen}(n+1) \frac{\pi}{2}}{(\rho+1)^{2}}+ \\
& +\frac{\cos (n+1) \frac{\pi}{2}}{p+1}\left(0-\frac{\pi}{2}\right) \\
& \frac{\operatorname{sen}\left[(p-1) \theta-\frac{\pi}{4}\right]}{(p-1)^{2}}=-\int_{\frac{\pi}{2}}^{\theta} d \theta \int_{\frac{\pi}{2}}^{\theta} \operatorname{sen}\left[(\rho-1) \theta-\frac{\pi}{4}\right] d \theta-\frac{\operatorname{sen}(n+1) \frac{\pi}{2}}{(p-1)^{2}}- \\
& -\frac{\cos (n+1) \frac{\pi}{2}}{p-1}\left(0-\frac{\pi}{2}\right)
\end{aligned}
$$


quindi sostituendo nella formola precedente, e avendo riguardo alla formola (1) e alla circostanza che quelle serie, che oltre ad avere il $\sqrt{n}$ al denominatore hanno anche un altro divisore che è almeno di second'ordine, come $(\rho \pm 1)^{2}$, $n(\rho \pm 1), \ldots$, sono tutte convergenti, mentre invece si è incerti sulla convergenza della serie $\mathbf{\Sigma} \frac{A_{n}}{\sqrt{2 n \pi}} \cos (n+1) \frac{\pi}{2}\left(\frac{1}{\rho+1}+\frac{1}{\rho-1}\right)$, si avrà :

$$
\mathbf{\Sigma} A_{n}\left\{\int_{\frac{\pi}{2}}^{\theta} d \theta \int_{\frac{\pi}{2}}^{\theta} X_{n} \operatorname{sen}^{\frac{3}{2}} \theta d \theta-\frac{\cos (n+1) \frac{\pi}{2}}{\sqrt{2 n \pi}}\left(\frac{1}{p+1}+\frac{1}{p-1}\right)\left(\theta-\frac{\pi}{2}\right)\right\}=c_{1} \theta+c_{1}^{\prime} ;
$$

e siccome $\frac{1}{p+1}+\frac{1}{p-1}=\frac{2}{n+1}+\frac{c_{n}}{n^{2}}$ ove $c_{n}$ è sempre inferiore a un numero finito, e il secondo membro di questa eguaglianza deve annullarsi per $\theta=\frac{\pi}{2}$ come si annulla il primo, si potrà anche scrivere:

$$
\Sigma A_{n}\left\{\int_{\frac{\pi}{2}}^{\theta} d \theta \int_{\frac{\pi}{2}}^{\theta} X_{n} \operatorname{sen}^{\frac{3}{2}} \theta d \theta-\frac{2 \cos (n+1) \frac{\pi}{2}}{(n+1) \sqrt{2 n \pi}}\left(\theta-\frac{\pi}{2}\right)\right\}=c_{2}\left(\theta-\frac{\pi}{2}\right),
$$

ove $c_{2}$ è una nuova costante; e alla serie del primo membro potrà ancora applicarsi una o più volte l'integrazione definita termine a termine in ogni intervallo compreso fra $\varepsilon$ e $\pi-\varepsilon$.

Si osservi ora che quando $\theta$ è compreso fra $\varepsilon$ e $\pi-\varepsilon$ con una doppia integrazione per parti si trova:

$$
\begin{aligned}
& \int_{\frac{\pi}{2}}^{\theta} X_{n} \operatorname{sen} \theta d \theta=\frac{1}{\sqrt{\operatorname{sen} \theta}} \int_{\frac{\pi}{2}}^{\theta} X_{n} \operatorname{sen}^{\frac{3}{2}} \theta d \theta-\left(\frac{1}{\sqrt{\operatorname{sen} \theta}}\right)_{\frac{\pi}{2}}^{l} \int_{\frac{\pi}{2}}^{\theta} d \theta \int_{\frac{\pi}{2}}^{\theta} X_{n} \operatorname{sen}^{\frac{3}{2}} \theta d \theta+ \\
& +\int_{\frac{\pi}{2}}^{\theta}\left(\frac{1}{\sqrt{\operatorname{sen} \theta}}\right)^{\prime \prime} d \theta \int_{\frac{\pi}{2}}^{\theta} d \theta \int_{\frac{\pi}{2}}^{\theta} X_{n} \operatorname{sen}^{\frac{3}{2}} \theta d \theta, \\
& \int_{\frac{\pi}{2}}^{\theta} \operatorname{sen} \theta d \theta \int_{\frac{\pi}{2}}^{\theta} X_{n} \operatorname{sen} \theta d \theta=\sqrt{\operatorname{sen} \theta} \int_{\frac{\pi}{2}}^{\theta} d \theta \int_{\frac{\pi}{2}}^{\theta} X_{n} \operatorname{sen}^{\frac{3}{2}} \theta d \theta+ \\
& +\int_{\frac{\pi}{2}}^{\theta} \operatorname{sen} \theta d \theta \int_{\frac{\pi}{2}}^{\theta}\left(\frac{1}{\sqrt{\operatorname{sen} \theta}}\right)^{\prime \prime} d \theta \int_{\frac{\pi}{2}}^{\theta} \int_{\frac{\pi}{2}}^{\theta} X_{n} \operatorname{sen}^{\frac{3}{2}} \theta d \theta
\end{aligned}
$$


e quindi sostituendo si avrà :

$$
\begin{gathered}
\mathbf{\Sigma} A_{n} \int_{\frac{\pi}{2}}^{\theta} \operatorname{sen} \theta d \theta \int_{\frac{\pi}{2}}^{\theta} X_{n} \operatorname{sen} \theta d \theta-\int_{\frac{\pi}{2}}^{\theta} \operatorname{sen} \theta d \theta \int_{\frac{\pi}{2}}^{\theta}\left(\frac{1}{\sqrt{\operatorname{sen} \theta}}\right)^{\prime \prime} d \theta \int_{\frac{\pi}{2}}^{\theta} d \theta \int_{\frac{\pi}{2}}^{\theta} X_{n} \operatorname{sen}^{\frac{3}{2}} \theta d \theta- \\
-\frac{2 \cos (n+1) \frac{\pi}{2}}{(n+1) \sqrt{2 n \pi}}\left(\theta-\frac{\pi}{2}\right) \sqrt{\operatorname{sen} \theta}=c_{2}\left(\theta-\frac{\pi}{2}\right) \sqrt{\operatorname{sen} \theta}
\end{gathered}
$$

e poichè l'essere applicabile una o più volte l'integrazione definita termine a termine alla serie del primo membro della formola (2), in ogni intervallo fra $\varepsilon$ e $\pi-\varepsilon$, porta che si abbia:

$$
\begin{gathered}
\mathbf{\Sigma} A_{n}\left\{\int_{\frac{\pi}{2}}^{\theta} \operatorname{sen} \theta d \theta \int_{\frac{\pi}{2}}^{\theta}\left(\frac{1}{\sqrt{\operatorname{sen} \theta}}\right)^{\prime \prime} d \theta \int_{\frac{\pi}{2}}^{\theta} d \theta \int_{\frac{\pi}{2}}^{\theta} X_{n} \operatorname{sen}^{\frac{3}{2}} \theta d \theta-\right. \\
\left.-\frac{2 \cos (n+1) \frac{\pi}{2}}{(n+1) \sqrt{2 n \pi}} \int_{\frac{\pi}{2}}^{\theta} \operatorname{sen} \theta d \theta \int_{\frac{\pi}{2}}^{\theta}\left(\frac{1}{\sqrt{\operatorname{sen} \theta}}\right)^{\prime \prime}\left(\theta-\frac{\pi}{2}\right) d \theta\right\}= \\
=c_{2} \int_{\frac{\pi}{2}}^{\theta} \operatorname{sen} \theta d \theta \int_{\frac{\pi}{2}}^{\theta}\left(\frac{1}{\sqrt{\operatorname{sen} \theta}}\right)^{\prime \prime}\left(\theta-\frac{\pi}{2}\right) d \theta
\end{gathered}
$$

e d'altra parte si ha anche:

$$
\begin{aligned}
& \int_{\frac{\pi}{2}}^{\theta}\left(\frac{1}{\sqrt{\operatorname{sen} \theta}}\right)^{\prime \prime}\left(\theta-\frac{\pi}{2}\right) d \theta=\left(\frac{1}{\sqrt{\operatorname{sen} \theta}}\right)^{\prime}\left(\theta-\frac{\pi}{2}\right)-\frac{1}{\sqrt{\operatorname{sen} \theta}}+1 \\
& \int_{\frac{\pi}{2}}^{\theta} \operatorname{sen} \theta \int_{\frac{\pi}{2}}^{\theta}\left(\frac{1}{\sqrt{\operatorname{sen} \theta}}\right)^{\prime \prime}\left(\theta-\frac{\pi}{2}\right) d \theta=-\sqrt{\operatorname{sen} \theta}\left(\theta-\frac{\pi}{2}\right)-\cos \theta
\end{aligned}
$$

sostituendo si troverà:

$$
\mathbf{\Sigma} A_{n}\left\{\int_{\frac{\pi}{2}}^{\theta} \operatorname{sen} \theta d \theta \int_{\frac{\pi}{2}}^{\theta} X_{n} \operatorname{sen} \theta d \theta+\frac{2 \cos (n+1) \frac{\pi}{2}}{(n+1) \sqrt{2 n \pi}} \cos \theta\right\}=-c_{2} \cos \theta
$$


0 anche, riponendo $\cos \theta=x$ :

$$
\Sigma A_{n}\left\{\int_{0}^{x} d x \int_{0}^{x} X_{n} d x+\frac{2 \cos (n+1)^{\frac{\pi}{2}}}{(n+1) \sqrt{2 n \pi}} x\right\}=-c_{2} x
$$

e questa formola varrà per tutti i valori di $x$ fra -1 e 1 , esclusi soltanto i valori 1 e -1 .

Ora, per $n>0$ si ha:

$$
\int_{0}^{x} X_{n} d x=\frac{X_{n+1}-X_{n-1}}{2 n+1}-\frac{X_{n+1}^{0}-X_{n-1}^{0}}{2 n+1}
$$

0 anche:

$$
\int_{0}^{x} X_{n} d x=\frac{X_{n+1}-X_{n-1}}{2 n+1}+\frac{X^{0}{ }_{n-1}}{n+1}
$$

ove $X_{n-1}^{0}$ e $X_{n+1}^{0}$ sono i valore di $X_{n-1}$ e $X_{n+1}$ per $x=0$, giacchè per $x=0$ da una formula nota si ha:

$$
(n+1) X_{n+1}^{0}+n X_{n-1}^{0}=0 ;
$$

quindi per $n>1$ si avrà :

$$
\begin{gathered}
\int_{0}^{x} d x \int_{0}^{x} X_{n} d x=\frac{X_{n+2}-X_{n}}{(2 n+1)(2 n+3)}-\frac{X_{i 2}-X_{n-2}}{(2 n-1)(2 n+1)}+ \\
+\frac{X_{n}^{0}}{(2 n+1)(n+2)}-\frac{X_{n-2}^{0}}{(2 n+1) n}+\frac{X_{n}^{J} 1}{n+1} x,
\end{gathered}
$$

e per $n=1$ sarà invece:

$$
\int_{0}^{x} d x \int_{0}^{x} X_{1} d x=\frac{X_{3}-X_{1}}{3.5}+\frac{x}{6}
$$

e ora, poiche dalla formola (1) si ha facilmente:

$$
\frac{X_{n-1}^{0}}{n+1}=-\frac{2 \cos (n+1) \frac{\pi}{2}}{(n+1) \sqrt{2 n \pi}}+\frac{b_{n}}{n^{2} \sqrt{n}},
$$

ove $b_{n}$ col crescere indefinito di $n$ resta sempre inferiore a un numero finito, e inoltre le due serie $\mathbf{\sum} \frac{A_{n} b_{n} x}{n^{2} \sqrt{n}}, \mathbf{\sum} A_{n}\left(\frac{X_{n}^{0}}{(2 n+1)(n+2)}-\frac{X_{n-2}}{(2 n+1) n}\right)$ sono convergenti, sostituendo nella formola (3) si troverà subito la seguente:

$$
A_{0} \frac{x^{9}}{2}+\frac{A_{1}\left(X_{3}-X_{1}\right)}{3 \cdot 5}+\sum_{2}^{\infty} A_{n}\left\{\frac{X_{n+2}-X_{n}}{(2 n+1)(2 n+3)}-\frac{X_{n}-X_{n-2}}{(2 n-1)(2 n+1)}\right\}=c_{8} x+c_{4} \text {, }
$$


ove $c_{3}$ e $c_{4}$ sono due nuove costanti; e questa formola, sebbene dimostrata pei soli valori di $x$ fra -1 e 1 esclusi i valori \pm 1 , varrà anche a questi limiti, giacche la serie del primo membro e tutti gli altri termini sono funzioni continue di $x$.

Sussistendo ora questa formola anche per $x= \pm 1$, si avrà $\frac{A_{0}}{2}=c_{3}+c_{4}$, e $\frac{A_{0}}{2}=-c_{3}+c_{4} ;$ quindi sarà $c_{3}=0, c_{4}=\frac{A_{0}}{2}$, e perciò si avrà :

$$
A_{0}\left(\frac{x^{2}}{2}-\frac{1}{2}\right)+\frac{A_{1}\left(X_{3}-X_{1}\right)}{3 \cdot 5}+\sum_{2}^{\infty} A_{n}\left\{\frac{X_{n+2}-X_{n}}{(2 n+1)(2 n+3)}-\frac{X_{n}-X_{n-2}}{(2 n-1)(2 n+1)}\right\}=0
$$

ovvero:

$$
\frac{A_{0}\left(X_{3}-X_{0}\right)}{3}+\frac{A_{1}\left(X_{3}-X_{1}\right)}{3 \cdot 5}+\sum_{2}^{\infty} A_{n}\left\{\frac{X_{n+2}-X_{n}}{(2 n+1)(2 n+3)}-\frac{X_{n}-X_{n-2}}{(2 n-1)(2 n+1)}\right\}=0
$$

yiacchè $\frac{x^{2}}{2}-\frac{1}{2}=\frac{X_{2}-X_{0}}{3}$.

Osservando ora che, siccome la serie del primo membro è convergente in ugual grado nell'intervallo da -1 a 1 , si può applicarle l'integrazione definita termine a termine da -1 a 1 dopo averla moltiplicata tutta per $X_{m}$; si troverà subito che i coefficienti di ogni funzione $X_{n}$ nel primo membro della formola precedente devono essere tutti nulli, e si avranno quindi i due sistemi di equazioni seguenti:

$$
\begin{gathered}
-\frac{A_{0}}{3}+\frac{A_{2}}{3 \cdot 5}=0, \\
\frac{A_{0}}{1 \cdot 3}-\frac{A_{2}}{3 \cdot 5}-\frac{A_{2}}{5 \cdot 7}+\frac{A_{4}}{7 \cdot 9}=0, \\
\frac{A_{2}}{5 \cdot 7}-\frac{A_{4}}{7 \cdot 9}-\frac{A_{4}}{9 \cdot 11}+\frac{A_{6}}{11 \cdot 13}=0, \\
\ldots \ldots \\
\frac{A_{2 n-2}}{(4 n-7)(4 n-5)}-\frac{A_{2 n \cdot 2}}{(4 n-5)(4 n-3)}-\frac{A_{2 n}}{(4 n-3)(4 n-1)}+\frac{A_{2 n}}{(4 n-1)(4 n+1)}=0, \\
\frac{A_{2 n}}{(4 n-3)(4 n-1)}-\frac{A_{2 n+2}}{(4 n-1)(4 n+1)}-\frac{A_{2 n 2}}{(4 n+1)(4 n+3)}+\frac{A_{1}}{(4 n+3)(4 n+5)}=0, \\
-\frac{A_{3}}{3 \cdot 5}+\frac{1}{5 \cdot 7}=0,
\end{gathered}
$$




$$
\begin{gathered}
\frac{A_{1}}{3 \cdot 5}-\frac{A_{3}}{5 \cdot 7}-\frac{A_{3}}{7 \cdot 9}+\frac{A_{5}}{9 \cdot 11}=0, \\
\frac{A_{3}}{7 \cdot 9}-\frac{A_{5}}{9 \cdot 11}-\frac{A_{5}}{11 \cdot 13}+\frac{A_{7}}{13 \cdot 15}=0, \\
\ldots \ldots \ldots \\
\frac{A_{2 n-5}}{(4 n-9)(4 n-7)}-\frac{A_{2 n-3}}{(4 n-7)(4 n-5)}-\frac{A_{2 n-3}}{(4 n-5)(4 n-3)}+\frac{A_{2 n-1}}{(4 n-3)(4 n-1)}=0, \\
\frac{A_{2 n-3}}{(4 n-5)(4 n-3)}-\frac{A_{2 n-1}}{(4 n-3)(4 n-1)}-\frac{A_{2 n-1}}{(4 n+1)(4 n+3)}+\frac{A_{2 n+1}}{(4 n+3)(4 n+5)}=0,
\end{gathered}
$$

le quali sommate respettivamente conducono alle altre:

$$
\frac{A_{2 n+2}}{4 n+5}=\frac{A_{2 n}}{4 n+1}, \quad \frac{A_{2 n+1}}{4 n+3}=\frac{A_{2 n-1}}{4 n-1},
$$

che valgono per tutti i valori di $n$, e quindi ci danno:

$$
A_{2^{n}}=(4 n+1) A_{0}, \quad A_{2^{n}+1}=(4 n+3) \frac{A_{1}}{3} \text {; }
$$

e ora, poichè di qui risulta che, se $A_{0}$ e $A_{1}$ non fossero zero, le quantità $A_{n}$ non sarebbero tali che i rapporti $\frac{A_{n}}{\sqrt{n}}$ tendano a zero col crescere indefinito di $n$, perchè esse diverrebbero infinite di prim'ordine insieme con $n$, si conclude che $A_{0}$ e $A_{1}$ e così tutte le $A_{n}$ devono essere identicamente nulle, e cosi il teorema enunciato in principio resta completamente dimostrato.

Pisa, 7 dicembre 1873. 